\title{
Analysis on Innovative Path of Lacquer Products in the Context of Fashion
}

\author{
Jing Chen ${ }^{1, *}$ \\ ${ }^{1}$ Fine Arts and Design College, Minjiang University, Fuzhou, Fujian 350001, China \\ *Corresponding author.Email: 39600466@qq.com
}

\begin{abstract}
Against the background that traditional lacquer art is difficult to adapt to the new life style and new fashion of the times, this paper, based on the connection of design and technology, takes "fashion" as the research object, explores the way to make lacquer art return to life through fashionable design with a sense of the times, and establishes the method and way to turn lacquer art into fashionable products under the new values, with a view to passing it on. The inheritance and development of traditional lacquer art in the contemporary social background provides ideas.
\end{abstract}

Keywords: Lacquer art, Fashion, Culture, Innovation.

\section{INTRODUCTION}

Lacquer art has experienced more than 7,000 years of inheritance and development in China. Lacquer ware used to be the daily life utensils used by the public at weddings or funerals as well as daily utensils. However, the vast majority of modern lacquer ware is only used for viewing, so that the contemporary lacquer art culture cannot be integrated into the field of public life. In the context that the form, function, pattern and color of lacquer products cannot adapt to the new way of life and fashion of new times, the research on the relationship between modern lacquer art products and fashion as well as the path of fusion innovation is of positive significance to explore the modern inheritance and innovation of lacquer art.

\section{THE DEFINITION OF TRADITIONAL CRAFT, DESIGN AND FASHION AND THEIR RELATION}

The dictionary defines "fashion" as "a popular trend", which contains two elements of the current time and prevalence [1]. After defining the concept of fashion, the relationship between tradition and fashion can be clarified. "Tradition" and "fashion"

*Fund: 2018 Young and Middle-aged Teacher Education Research Project in Fujian Province (JAS180360); Key Research Project of Minjiang University in 2018 (MYS18026) are not opposites. With a look back at the history, it can be seen that the classic lacquer ware, such as the cinnabar gold and wood carving furniture, red lacquer bowls, and red lacquer baskets and so on, are all products in line with the aesthetic taste of the public at that time, which are closely connected with the lifestyle of that time. Tradition is based on history, while fashion is based on contemporary times. Tradition is a history that has a living origin and walks into modern times. Tradition does not die out because of modern fashion and each period in history has its own unique fashion culture.

On the one hand, Chinese traditional craft culture lives in the present with an open vision, relying on fashionable carriers and modern design language. On the other hand, if fashion can fully understand and convey the image of craft culture, it can be nourished by traditional culture and become a classic. Fashion is rooted in culture. China's unique and rich craft and cultural resources provide more creative resources for the fashion industry, allowing fashion to show more breadth and depth. Design and fashion need the support of culture in order to create works with traditional connotations. The evolution from tradition to fashion needs to refer to contemporary aesthetics and lifestyles and advance through innovation in design. In short, fashion culture leads to different design directions. Design points out the development direction of traditional craft, and at the same time, design 
creates new fashion [2]. It is necessary to find a new connection between tradition and fashion and promote the sustainable development and utilization of traditional cultural resources. In the stage of vigorously carrying forward the excellent Chinese traditional culture and reshaping the confidence of the traditional culture of the Chinese nation today, it will be promising to introduce traditional crafts into the development of the fashion industry.

\section{TRADITIONAL TECHNOLOGY INNOVATION AND DEVELOPMENT OF THE CONCEPT OF FASHION}

In the aspect of modern inheritance of traditional craft, Japan pays attention to introducing traditional craft and culture into fashion life. In his book "Made with Heart and Soul", Toshiyuki Kita put forward the concept of "future design comes from traditional craft", showing that traditional lacquerware enters modern life through design, revealing a new way for Chinese lacquerware to create traditional culture through design and bring tradition into the present and future [3].

In "Design Research: Design for National Identity and People's Livelihood", Li Yanzu elaborated a new concept - to transform traditional Chinese craft into products with high sense of and quality and design, so that China's simple aesthetics for daily use and traditional craft culture can be rejuvenated in modern design and continue cultural memory [4]. In "The Language of Things", Deyan Sudjic proposed that the foundation of fashion lies in culture, which connects traditional culture, design and fashion, so that the traditional craft culture develops into a fashion craft atmosphere closely related to life through the introduction of design.

In the market, the integration of fashion brands and traditional crafts is becoming increasingly rich. "Shang Xia", part of the luxury brand Hermes Group, is dedicated to inheriting Chinese aesthetic culture and handmade crafts. It re-thinks them in the context of current life, and transforms them into "art" and "use" filled with breath of life of the times. Louis Vuitton invited the designer Chen Louis Vuitton to customize the "Tea Journey" hard boxes for tea ceremony. The overall design deeply excavates the Chinese classical traditional culture, and integrates the interpretation and expression of Western culture at the same time, blends Eastern culture and Western aesthetics, endows the work with the design feelings of "East sentiment with West appeal".

To sum up, by the link between design and craft, the concept of craft fashion can be used to expand the new path of lacquer art innovation and development. The cultural attribute, artistic attribute and natural attribute of lacquer art are the elements that can be imported in modern design, which fit the natural health, sense of culture, sense of art, quality and individualization that fashion life pursues. While adhering to Chinese lacquer materials, inheriting traditional craft culture and concreting unique Oriental aesthetic charm, it is necessary to integrate original personality and aesthetic elements of modern fashion life and inject humanistic care into the creation and application of products. The concept of craft fashion will enable lacquer art to establish the method and approach of turning to fashion products close to life in the context of modern culture, and open up a new vision of lacquer art innovation and development in a broader cross-field and interdisciplinary vision.

\section{ANALYSIS ON THE FASHION DESIGN PATH OF LACQUER PRODUCTS}

Through the comparative analysis of traditional lacquer art and modern fashion, the research idea of this paper is to find the converging interests between the two and explore the development path, so that the traditional lacquer art elements can live in the contemporary era in a fashionable way and integrate into the daily life. Nowadays, people have experienced and accepted the impact of multiculturalism on their life, and they have different demands for life. In line with the fact that people pursue natural health, sense of culture, sense of art, quality, personalization as their life goals, the core of making Chinese traditional lacquer art fashionable lies in its natural lacquer material, lacquer language and lacquer quality. Therefore, it is an effective way to realize the fashion of lacquer art to find out the natural language, cultural language and artistic language in line with the modern aesthetic concept from the lacquer art, and to integrate it into the modern life through the modern design means and language expression and expansion, and to find a new way to develop and utilize the resources of lacquer art. 


\subsection{Taking in the Beauty of Lacquer Art Culture}

Chinese lacquer art culture is an extension of China's original creation art, with oriental connotations lie mystery, nobleness and elegance, and the pursuit of the unity of people, objects and environment, and it advocates the harmonious coexistence of implements. In the fashion creation of lacquer art, it is necessary to understand the essence of traditional lacquer art, dig deep into the cultural view of lacquer, and express designers' design ideas through the medium of lacquer perceptually, so as to meet the pursuit of modern fashion life for cultural sense. Toshiyuki Kita believes that western design tends to emphasize functionality while neglecting the soul and temperature in products, and the materials and techniques of traditional lacquer art can just make up for this deficiency [3] $]^{17}$. For example, the lacquer style watch in the Credor series under SEIKO (see "Figure 1") is designed with careful screening and color matching by the world class lacquer art master Quan Longfu, who presented to the public with exquisite painting art interpretation of modern personality fashion by applying the traditional Japanese lacquer technique to the dial of the watch, in combination of the techniques of mother-of-pearl and maki-e. Jewelry brand Van Cleef \& Arpels cooperated with Japanese lacquer master Junichi Hakose to develop a series of lacquer painted butterfly brooches ("Figure 2"). They conform to no conventional pattern traditional Chinese lacquer both in terms of pattern and color, integrated with more fashionable meaning in addition to inheritance. The products are integrated into the spirit of the craftsman and the heart of the hand, so that the products show more cultural deposits and warmth, presenting the luxury and elegance with both eastern and western temperament.

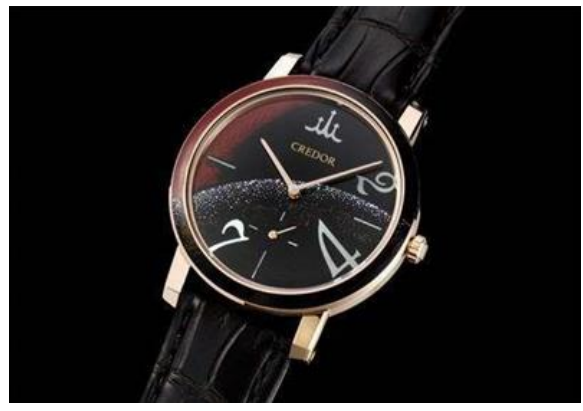

Figure 1 Lacquer watch.

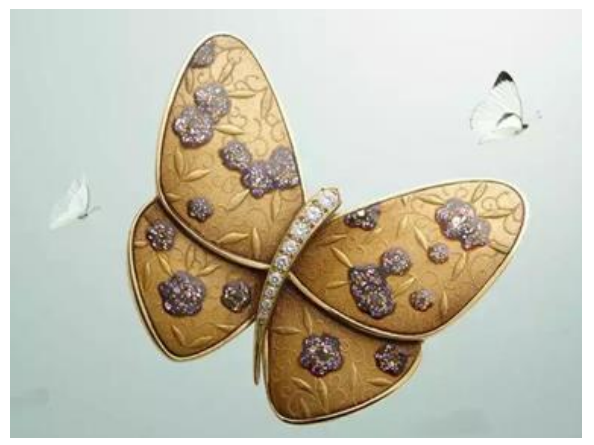

Figure 2 Lacquer painted butterfly brooch.

\subsection{Broadening the Application Field of Lacquer Art}

\subsubsection{Crossover Cooperation with Different Fields}

In the context of cultural consumption, product has been beyond its original scope. The crossover between traditional lacquer ware with other functional products or fashion themes will see more market space for development and use. It will become an important direction for the traditional lacquer fashion to introduce the concept of "crossover" to the design of lacquer art. Through crossover cooperation, lacquer art can provide creative expression ways and source of inspiration for other fields of design, which can meanwhile display the cultural flavor, vision elements and moulding rules flexibly through modern design means, by understanding the core elements of traditional lacquer art [4].

Integrated innovation can be explored between traditional lacquer art with packaging design, jewelry design, industrial product design, furniture design, indoor setting design, cultural tourism and sculpture, etc. it can be modern technology products like information device and phone case, etc., or ornaments, decorations and bags, etc. developed with fashion as the design idea. For example, the bag brand GROTTO made an exploration of integrating lacquer art and fashionable bags, which is a refreshing trial; the Yangzhou Lacquer Factory and the School of Design, Nanjing University of the Arts have jointly developed the $U$ disk series and brooch series of lacquer art gifts for Shanghai World Expo, which are combined with the lacquer techniques such as conch staining, gold size and lacquer engraving. With fancy shapes, strong personality and interesting connotation, the products have become 
the trend of fashion and meet the consumers' pursuit of individuation, fashion and emotion.

\subsubsection{Reflection of the Change of Language in Accordance with the Times}

The involvement of lacquer art in life depends on the uniqueness of its materials. The beauty of its material and texture is a major advantage for the expression language of lacquer art. By use of techniques such as lacquer engraving, lacquer embossing, gold and silver inlay, jin xipi (a kind of method to process lacquer with gold foil) to shape texture, lacquer art can meet the pursuit of delicacy and quality of modern fashion life, which is an important entry point for traditional lacquer art to get involved in modern fashion life.

At the same time, the diversified aesthetic has put forward new requirements for the creation techniques of lacquer art. The diversified expression forms of lacquer materials can expand the expressive force of lacquer materials through different stimulation and creation, and build a broader formal language:

\subsubsection{Traditional Pattern Innovation}

It is necessary to redesign traditional patterns, seek changes in invariability and give the product a new form of expression through transplant, induction and refining technique and with modern expression language and adornment pattern, to meet the modern people's demand for living quality and taste.

\subsubsection{Combination with Other Materials}

Integrating lacquer materials with bamboo, ceramics, metal, glass and leather, etc., as well as 3D printing technology, can provide ideas for design ("Figure 3") and open up thinking. Taking the lacquered pottery cup series by Wang Yue as an example, the combination of pottery and lacquer is used to create a batch of new tea ceremony utensils. With ceramic as the main material of tire base and lacquer as the main veneer, the high temperature resistance of ceramic materials is perfectly combined with the mysterious and elegant visual effect of lacquer materials and the jade-like touch of lacquer materials ("Figure 4").

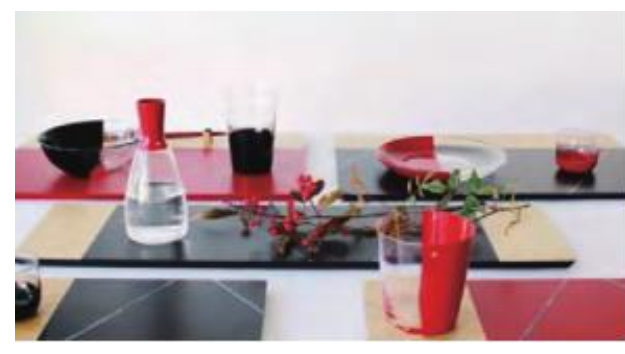

Figure 3 The combination of lacquer and glass.

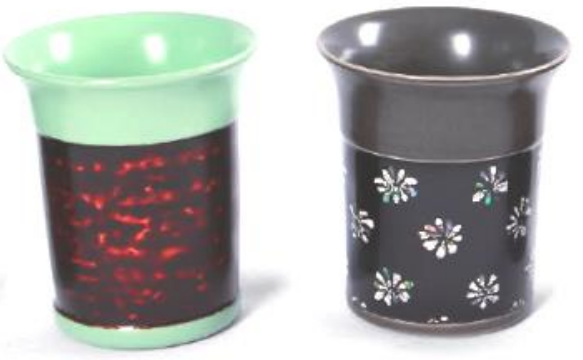

Figure 4 Lacquered pottery cup.

\subsubsection{Functional Domain Extension}

The traditional lacquer material itself is a natural and environmentally friendly material. After drying, the lacquer is waterproof, easy to be washed, light in volume, heat-insulated and wearresisting, and diverse in variety, all making it a very ideal daily necessities and high-quality decorative medium. The Japanese are constantly expanding and exploring the application of lacquerware. At present, the function of lacquerware is extended to the field of medical care and health care, and health care products with sterilization and heat preservation functions are developed in Japan [5].

\subsubsection{Giving Play to the Unique Tactility and Interactivity of Lacquer Materials}

The world is moving towards a way of life that coexists with nature. Lacquer art products give off a natural feeling of recovering our original simplicity in use and each touch of the hand is the polishing of the lacquer art works. The cumulative use of the hand over a long period of time will gradually reveal this kind of interaction, increasingly revealing a kind of implicit beauty, which skillfully interacts with the language of perception, touch and texture to create a sense of beauty that resonates with users. The unique temperament and spirituality corresponds to the emotional needs of modern people with fashion life.

Based on the unique characteristics of lacquer, more carriers can be found for lacquer art adopted 
in a small range of products. It can play the role of finishing touch, and penetrate into people's daily life. For example, the handle of vacuum cleaners can be processed by use of lacquer art. Lacquer materials can be decorated in the power switch panel, the handle in central control of cars so that users will feel comfortable, and the protection and decorative function of lacquer art can be played.

\subsubsection{Updating the Concept of Lacquer Art Creation}

In the new era, lacquer artists are required to have new ideas and improve their creative ability, pay more attention to the characteristics of lacquer art of the times and the exploration of the relationship between lacquer art and the development and evolution of the times. Attention should be paid to the balance between "art" and "use" in lacquer art. Based on the study of modern people's aesthetic and life style, the inheritance of traditional lacquer art must meet the aesthetic needs of modern people, and be closely related to the thoughts and feelings of modern people. $\mathrm{Yu}$ Zheng's "Traditional Handicrafts and Modern Life the New Context of Lacquer Art" and Ma Hang's "Lacquer Ware, Life and Attitude" show that the design style of lacquer art products is closely related to the development of the times, reflecting the evolution of people's demand for lacquer art products in different times. Japanese lacquer artists developed lacquered forks, trays, beer glasses, wine glasses, coffee sets and many other tableware have developed the dining form of mixing and matching in line with things emerging in modern times, using wood as the body and decorating it with lacquer [5]. It is the key for traditional lacquer art to get involved in fashion life to grasp the spirit of the times and fully express lacquer materials and techniques.

\section{THE CORRELATION OF FASHIONABLE PRODUCTS OF LACQUER ART AND MARKET}

In addition to innovation factors, the development of lacquer art is limited by its complicated production technology, low production efficiency and expensive lacquer materials. Therefore, in order to let the lacquer art into a broader field of social production and life, it is necessary consider the relationship between lacquer art and machine production, batch manufacturing, marketing and mass consumption. According to the market and consumer demand, the market positioning is divided into: general daily use, cultural fashion, and personalized customization, with high-end and mass orientation lying parallel. Manual production and mechanized production coexist in different divisions and the system corresponds to different design, process decoration and manufacturing methods [6].

The acceptance of classic inherent image formed by traditional lacquer art by the public can never be achieved overnight, and the stratification and acceptance of users under the market environment should be fully considered. Fashion is always led by place of a few people, and the mass is the follower of fashion, so, fashion designs of lacquer art should consider the distinction of light spot and hotspot of contemporary consumer market. The hotspot consumer market is the product type that can be accepted by the public; the light-spot consumer market is the product type designed for the small number of people. Through the cooperation of design elites and craftsmen, it is necessary to promote market highlights, so as to promote the market development and form consumption hot spots. After repeated iteration and promotion, the breakthrough of qualitative change can be realized with quantitative accumulation, and the modern and fashionable product images will be accepted and loved by the public.

\section{CONCLUSION}

The development path of craft fashion integrates design, craft, brand, culture and market and other fields. It is necessary to conform to the lifestyle of the contemporary people, open the modern innovation and development of the traditional lacquer art in a natural way of design expression, and open the modern innovation and development of the traditional lacquer art in a fashion way, so that the traditional lacquer art culture and the fashion industry can find the appropriate meeting point. Only in this way can the integration of craft and fashion go deep into the daily life and enter the hearts of the people [7]. Only by dealing with the relationship between lacquer art and modern life fashion from the perspective of "utilization" and "development" can a way for the traditional lacquer art be created to return to the daily life of the public.

\section{AUTHORS' CONTRIBUTIONS} Chen.

This paper is independently completed by Jing 


\section{REFERENCES}

[1] Ci Hai. Shanghai: Shanghai Lexicographical Publishing House, 1989. (in Chinese)

[2] The Use of Fashion Language to Express Traditional Culture Is Also Trendy [N]. October 9, 2018, People's Daily, the third edition. (in Chinese)

[3] Toshiyuki Kita. Made with Heart and Soul [M]. Beijing: Publishing House of Electronics Industry, 2014: 63. (in Chinese)

[4] Li Yanzu. Contemporary and Regional Features of Traditional Arts and Crafts - on the Protection and Development of Traditional Arts and Crafts [J]. Journal of Nanjing Arts Institute (Fine Arts \& Design), 2008 (1). (in Chinese)

[5] Huang Junjie. Integration of Traditional Lacquer Art and Modern Design Exploration of Modern Lacquer Design [J]. Journal of Xiamen Academy of Arts and Crafts, Fuzhou University, 2014(6). (in Chinese)

[6] Yu Zheng. Traditional Handicrafts and Modern Life the New Context of Lacquer Art [J]. Sculpture, 2010 (5). (in Chinese)

[7] Li Yanzu. Design Research: Design for National Identity and People's Livelihood [M]. Chongqing: Chongqing University Press, 2010. 182-183. (in Chinese) 